\title{
Hadron structure in AdS/QCD
}

\author{
Valery E. Lyubovitskij ${ }^{* \dagger}$ \\ Institut für Theoretische Physik, Universität Tübingen, \\ Kepler Center for Astro and Particle Physics, \\ Auf der Morgenstelle 14, D-72076 Tübingen, Germany \\ E-mail: lubovit@tphys.physik.uni-tuebingen.de
}

\section{Thomas Gutsche}

Institut für Theoretische Physik, Universität Tübingen,

Kepler Center for Astro and Particle Physics,

Auf der Morgenstelle 14, D-72076 Tübingen, Germany

E-mail: thomas.gutscheduni-tuebingen.de

\section{Ivan Schmidt}

Departamento de Física y Centro Científico Tecnológico de Valparaíso (CCTVal), Universidad Técnica Federico Santa María, Casilla 110-V, Valparaíso, Chile

E-mail: ivan.schmidt@usm.cl

\section{Alfredo Vega}

Departamento de Física y Centro Científico Tecnológico de Valparaíso (CCTVal), Universidad Técnica Federico Santa María, Casilla 110-V, Valparaíso, Chile

E-mail: alfredo.vega@usm.cl

We perform a detailed analysis of nucleon electromagnetic and axial form factors in a holographic soft-wall model. Our approach is based on an action which describes hadrons with broken conformal invariance and incorporates confinement through the presence of a background dilaton field. For $N_{c}=3$ we describe the nucleon structure in a superposition of a three valence quark state with high Fock states including an adjustable number of partons (quarks, antiquarks and gluons) via studying the dynamics of 5D fermion fields of different scaling dimension in anti-de Sitter (ADS) space. According to the gauge/gravity duality the 5D fermion fields of different scaling dimension correspond to the Fock state components with a specific number of partons. In the present application we restrict to the contribution of 3,4 and 5 parton components in the nucleon Fock state. With a minimal number of free parameters (dilaton scale parameter, mixing parameters of partial contributions of Fock states, coupling constants in the effective Lagrangian) we achieve a reasonable agreement with data for the nucleon form factors.

The 7th International Workshop on Chiral Dynamics,

August 6 -10, 2012

Jefferson Lab, Newport News, Virginia, USA

\footnotetext{
*Speaker.

†n leave of absence from Department of Physics, Tomsk State University, 634050 Tomsk, Russia
} 


\section{Introduction}

Based on the gauge/gravity duality [1], a class of AdS/QCD approaches which model QCD by using methods of extra-dimensional field theories formulated in anti-de Sitter (AdS) space, was recently successfully developed for describing the phenomenology of hadronic properties. One of the popular formalisms of this kind is the "soft-wall" model [2], which uses a soft infrared (IR) cutoff in the fifth dimension. Here we present a detailed analysis of the nucleon electromagnetic form factors in a holographic soft-wall model [3]-[5] considering the inclusion of higher-dimensional fermion fields. Thus high-Fock state contributions are holographically incorporated in the nucleon. This novel approach is based on an action which describes hadrons with broken conformal invariance and which incorporates confinement through the presence of a background dilaton field. Notice the role of higher Fock components in the pion, in the context of holographic QCD, was considered before in Refs. [6]. In our framework for $N_{c}=3$ nucleons are considered as a superposition of three valence quark states and high Fock states including an adjustable number of partons (quarks, antiquarks and gluons) by studying the dynamics of the 5D fermion fields of different scaling dimension in AdS space. According to the gauge/gravity duality the 5D fermion fields of different scaling dimension correspond to Fock state components with a specific number of partons.

\section{Approach}

We consider the propagation of a fermion field $\Psi(x, z)$ with spin $J=1 / 2$ in 5-dimensional AdS space, which contains the contributions of different twist-dimensions. In the language of the AdS/QCD dictionary it corresponds to the inclusion of the three-quark and higher-parton states in the nucleon. For this first time we restrict ourselves to the contribution of $3 q, 3 q+g, 3 q+q \bar{q}$ and $3 q+2 g$ Fock states, where $q, \bar{q}$ and $g$ denote quark, antiquark and gluon, respectively. The main idea for describing the nucleon in AdS/QCD is based on the correspondence (see detailed discussion in Refs. [7]) between the spinor fields propagating in the bulk space and the QCD interpolating operators creating the nucleons on the boundary of AdS space. The appropriate boundary conditions for the bulk field on the boundary of AdS space ensure that such correspondence is precise due to the equivalence of the functional integrals of both the boundary and bulk theories. In particular, in the boundary theory (QCD) we define the left- and right-handed chiral doublets of nucleons $\mathscr{O}_{L}$ and $\mathscr{O}_{R}$. Since the chiral symmetry of the boundary theory is equivalent to the gauge symmetry in the bulk, we need to introduce the pair of bulk fermion fields $\Psi_{ \pm}(x, z)$, which are holographic analogues of the $\mathscr{O}_{R / L}$ operators. In particular, the bulk fields $\Psi_{ \pm}(x, z)$ contain important information about the baryon structure. On one side, their boundary values (non-normalizable solutions) are analogues of the sources for the QCD interpolating operators, which then via the evaluation of the Euclidean generating functionals produce the correlation functions of QCD operators. On the other side, these fields contain normalizable modes (these are regular and therefore are vanishing on the boundary) - profiles in extra dimension, which correspond to the baryon wave functions or expectation values of QCD operators. In our approach the conformal and chiral symmetries are spontaneously broken via the introduction of the background field (dilaton) $\varphi(z)$ in the effective action. We choose the quadratic dependence of the dilaton on the holographic coordinate $z$, i.e. $\varphi(z)=\kappa^{2} z^{2}$ with $\kappa$ being a free scale parameter, which scales as $\mathscr{O}\left(\sqrt{N_{c}}\right)$ in the large 
$N_{c}$-expansion. In particular, later we show that the nucleon (baryon) mass is proportional to the parameter $\kappa$, which is consistent with large $N_{c}$ QCD: $M_{N} \sim \kappa \sqrt{N_{c}} \sim N_{c}$. The main advantage of the dilaton with quadratic profile is the possibility to produce linear Regge-like trajectories for hadron masses. The relevant AdS/QCD action for the description of the nucleon electromagnetic and axial form factors is constructed in terms of the fermion fields $\Psi_{ \pm, \tau}(x, z)$ with spin $J=1 / 2$ and scaling dimension $\tau$, the vector field $V_{M}(x, z)$ with spin $J=1$ (holographic analogue of the electromagnetic field) and the axial field $A_{M}(x, z)$ (holographic analogue of the axial isovector field) [5]

$$
\begin{aligned}
S & =\int d^{4} x d z \sqrt{g} e^{-\varphi(z)}\left\{\mathscr{L}_{\Psi}(x, z)+\mathscr{L}_{V+A}(x, z)+\mathscr{L}_{\text {int }}(x, z)\right\}, \\
\mathscr{L}_{\Psi}(x, z) & =\sum_{i=+,-} \sum_{\tau} c_{\tau} \bar{\Psi}_{i, \tau}(x, z) \hat{\mathscr{D}}_{i}(z) \Psi_{i, \tau}(x, z), \\
\mathscr{L}_{V+A}(x, z) & =-\frac{1}{4} V_{M N}(x, z) V^{M N}(x, z)-\frac{1}{4} A_{M N}(x, z) A^{M N}(x, z), \\
\mathscr{L}_{\text {int }}(x, z) & =\sum_{i=+,-} \sum_{\tau} c_{\tau} \bar{\Psi}_{i, \tau}(x, z)\left\{\hat{\mathscr{V}}_{i}(x, z)+\hat{\mathscr{A}}_{i}(x, z)\right\} \Psi_{i, \tau}(x, z), \\
\hat{\mathscr{D}}_{ \pm}(z) & =\frac{i}{2} \Gamma^{M} \stackrel{\leftrightarrow}{\partial}_{M} \mp\left(\mu+U_{F}(z)\right), \quad A \stackrel{\leftrightarrow}{ } B \equiv A(\partial B)-(\partial A) B, \\
\hat{\mathscr{V}}_{ \pm}(x, z) & =Q_{N} \Gamma^{M} V_{M}(x, z) \pm \frac{i}{4} \eta_{V}\left[\Gamma^{M}, \Gamma^{N}\right] V_{M N}(x, z) \pm g_{V} \tau_{3} \Gamma^{M} i \Gamma^{z} V_{M}(x, z), \\
\hat{\mathscr{A}}_{ \pm}(x, z) & =\frac{\tau_{3}}{2}\left(\mp \Gamma^{M} A_{M}(x, z)+\frac{i}{4} \eta_{A}\left[\Gamma^{M}, \Gamma^{N}\right] A_{M N}(x, z)+g_{A} \Gamma^{M} i \Gamma^{z} A_{M}(x, z)\right) .
\end{aligned}
$$

Here $V(A)_{M N}$ is the stress tensor of the vector (axial) field, $Q_{N}$ and $\tau_{3}$ are nucleon charge and isospin matrices, $\Gamma^{M}$ are the five-dimensional Dirac matrices. $\mu$ is the bulk fermion mass related to the scaling dimension $\tau$ as $m=\mu R=\tau-3 / 2$. Notice that the scaling dimension of the AdS fermion field is holographically identified with the scaling dimension of the baryon interpolating operator $\tau=N+L$, where $N$ is the number of partons in the baryon and $L=\max \left|L_{z}\right|$ is the maximal value of the $z$-component of the quark orbital angular momentum in the light-front wavefunction [8]. $U_{F}(z)=\varphi(z) / R$ is the dilaton field dependent effective potential providing the correct asymptotics of the nucleon electromagnetic form factors at large $Q^{2}[9,[0]$. In this paper we restrict to the three leading contributions $\tau=3,4$ and 5. The coefficients $c_{\tau}$ are a set of parameters which take into account the mixing of AdS fermion fields with different scaling dimension $\tau$, which are constrained by the correct normalization of the kinetic term of the four-dimensional spinor field and by charge conservation as $\sum_{\tau} c_{\tau}=1$ [沛]. The five free parameters $\kappa, c_{3}, c_{4}, g_{V}$ and $\eta_{A}$ are fixed to the values $\kappa=383 \mathrm{MeV}, c_{3}=1.25, c_{4}=0.16, g_{V}=0.3, \eta_{A}=0.5$ [ [⿰]\zh26 .

\section{Results}

One of advantages of the soft-wall AdS/QCD model is that most of the calculations can be done analytically. E.g., the nucleon mass is given by the expression $M_{n}=2 \kappa \sum_{\tau} c_{\tau} \sqrt{n+\tau-1}$. As we stressed before, the nucleon (baryon) mass is proportional to the parameter $\kappa$ and this is consistent with large $N_{c}$ QCD: $M_{N} \sim \kappa \sqrt{\tau} \sim N_{c}$, where $\tau \sim N_{c}$. Next we present the numerical analysis of nucleon properties [5]. All analytical expressions are given in [5]. In Table I we present the results for the nucleon mass and the electroweak properties of nucleons. Selected results for the 
nucleon form factors in comparison to known data are shown in Figs. 1 and 2. We demonstrated that the soft-wall holographic model reproduces the main features of the electromagnetic structure of the nucleon. In particular, we achieved the following results: the analytical power scaling of the elastic nucleon form factors at large momentum transfers in accordance with quark-counting rules; reproduction of experimental data for magnetic moments and electromagnetic radii. One can see that with a minimal number of free parameters (five parameters) we obtain a reasonable description of the nucleon electromagnetic form factors including the correct power scaling at large $Q^{2}$. It demonstrates that the soft-wall model successfully describes nucleon structure at any resolution scale. In a next step, one can include effects of quark masses and extend the approach to nucleon resonances, light baryons with higher spins, strange and heavy baryons.

\section{Acknowledgments}

The authors thank Stan Brodsky, Guy de Téramond and Werner Vogelsang for useful discussions. This work was supported by the DFG under Contract No. LY 114/2-1, by Federal Targeted Program "Scientific and scientific-pedagogical personnel of innovative Russia" Contract No. 02.740.11.0238, by FONDECYT (Chile) under Grant No. 1100287. V. E. L. would like to thank Departamento de Física y Centro Científico Tecnológico de Valparaíso (CCTVal), Universidad Técnica Federico Santa María, Valparaíso, Chile for warm hospitality. A. V. acknowledges the financial support from FONDECYT (Chile) Grant No. 3100028.

\section{References}

[1] J. M. Maldacena, Adv. Theor. Math. Phys. 2, 231 (1998) [Int. J. Theor. Phys. 38, 1113 (1999)] S. S. Gubser, I. R. Klebanov and A. M. Polyakov, Phys. Lett. B 428, 105 (1998); E. Witten, Adv. Theor. Math. Phys. 2, 253 (1998).

[2] A. Karch, E. Katz, D. T. Son and M. A. Stephanov, Phys. Rev. D 74, 015005 (2006); S. J. Brodsky and G. F. de Teramond, Phys. Rev. Lett. 96, 201601 (2006); O. Andreev, Phys. Rev. D 73, 107901 (2006)

[3] A. Vega and I. Schmidt, Phys. Rev. D 78, 017703 (2008); Phys. Rev. D 79, 055003 (2009).

[4] T. Branz, T. Gutsche, V. E. Lyubovitskij, I. Schmidt and A. Vega, Phys. Rev. D 82, 074022 (2010); A. Vega, I. Schmidt, T. Branz, T. Gutsche and V. E. Lyubovitskij, Phys. Rev. D 80, 055014 (2009); T. Gutsche, V. E. Lyubovitskij, I. Schmidt and A. Vega, Phys. Rev. D 85, 076003 (2012).

[5] T. Gutsche, V. E. Lyubovitskij, I. Schmidt and A. Vega, Phys. Rev. D 86, 036007 (2012); A. Vega, I. Schmidt, T. Gutsche, V. E. Lyubovitskij, Phys. Rev. D 83, 036001 (2011).

[6] S. J. Brodsky, F. G. Cao and G. F. de Teramond, Phys. Rev. D 84, 075012 (2011). G. F. de Teramond and S. J. Brodsky, [arXiv:1203.4025 [hep-ph]].

[7] D. K. Hong, T. Inami and H. U. Yee, Phys. Lett. B 646, 165 (2007)

[8] S. J. Brodsky, G. F. de Teramond, Phys. Rev. Lett. 96, 201601 (2006); S. J. Brodsky and G. F. de Teramond, Phys. Rev. D 77, 056007 (2008).

[9] G. F. de Teramond and S. J. Brodsky, Nucl. Phys. Proc. Suppl. 199, 89 (2010); AIP Conf. Proc. 1432, 168 (2012).

[10] Z. Abidin and C. E. Carlson, Phys. Rev. D 79, 115003 (2009).

[11] S. J. Brodsky, arXiv:hep-ph/0208158. 
Table 1: Mass and electromagnetic properties of nucleons

\begin{tabular}{|c|c|c|}
\hline Quantity & Our results & Data \\
\hline$m_{p}$ (GeV) & 0.93827 & 0.93827 \\
\hline$\mu_{p}$ (in n.m.) & 2.793 & 2.793 \\
\hline$\mu_{n}$ (in n.m.) & -1.913 & -1.913 \\
\hline$g_{A}$ & 1.270 & 1.2701 \\
\hline$r_{E}^{p}$ (fm) & 0.840 & $0.8768 \pm 0.0069$ \\
\hline$\left\langle r_{E}^{2}\right\rangle^{n}\left(\mathrm{fm}^{2}\right)$ & -0.117 & $-0.1161 \pm 0.0022$ \\
\hline$r_{M}^{p}(\mathrm{fm})$ & 0.785 & $0.777 \pm 0.013 \pm 0.010$ \\
\hline$r_{M}^{n}(\mathrm{fm})$ & 0.792 & $0.862_{-0.008}^{+0.009}$ \\
\hline$r_{A}(\mathrm{fm})$ & 0.667 & $0.67 \pm 0.01$ \\
\hline
\end{tabular}
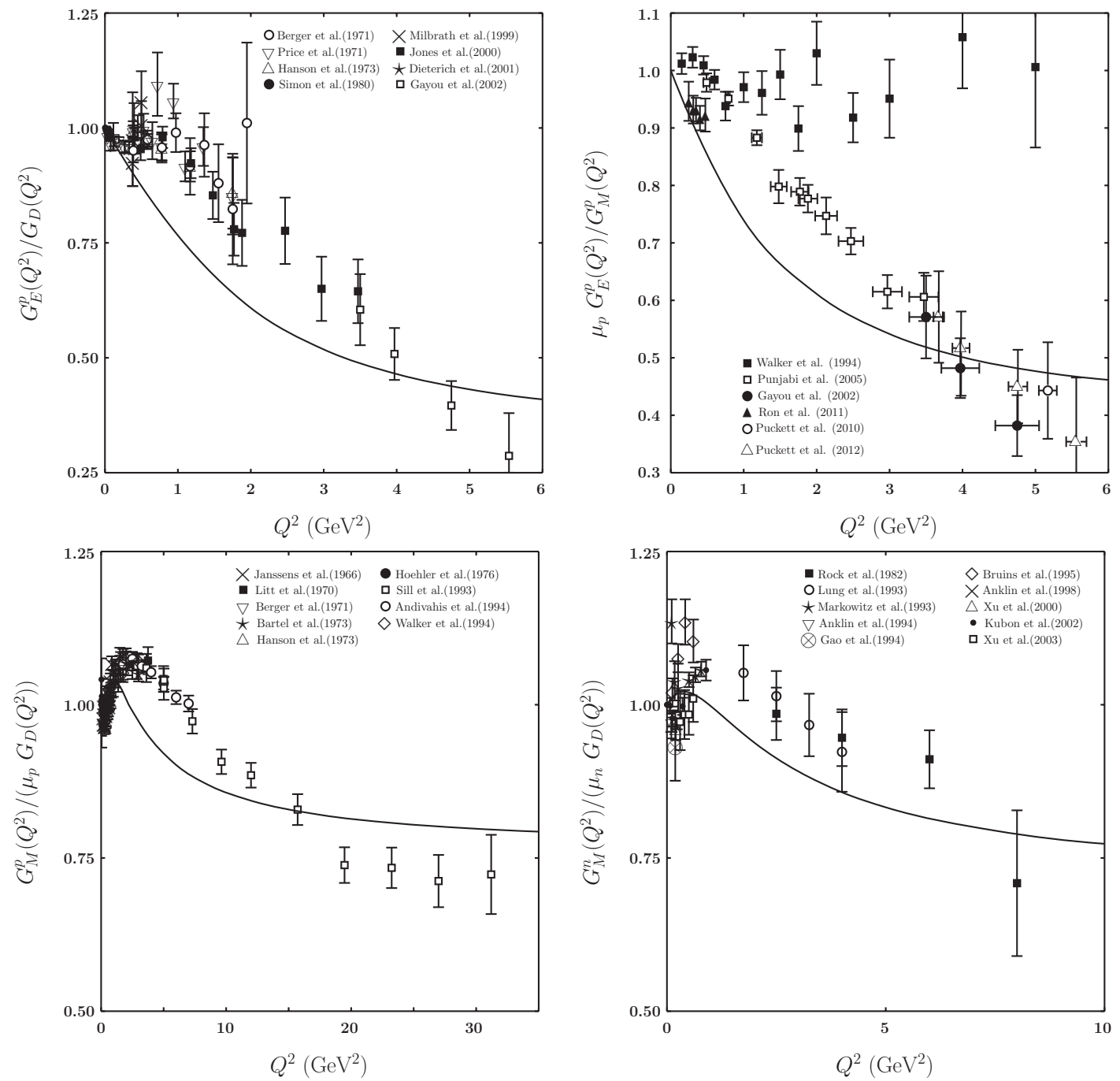

Figure 1: Ratios $G_{E}^{p}\left(Q^{2}\right) / G_{D}\left(Q^{2}\right), \mu_{p} G_{E}^{p}\left(Q^{2}\right) / G_{M}^{p}\left(Q^{2}\right), G_{M}^{p}\left(Q^{2}\right) /\left(\mu_{p} G_{D}\left(Q^{2}\right)\right)$ and $G_{M}^{n}\left(Q^{2}\right) /\left(\mu_{n} G_{D}\left(Q^{2}\right)\right)$. 

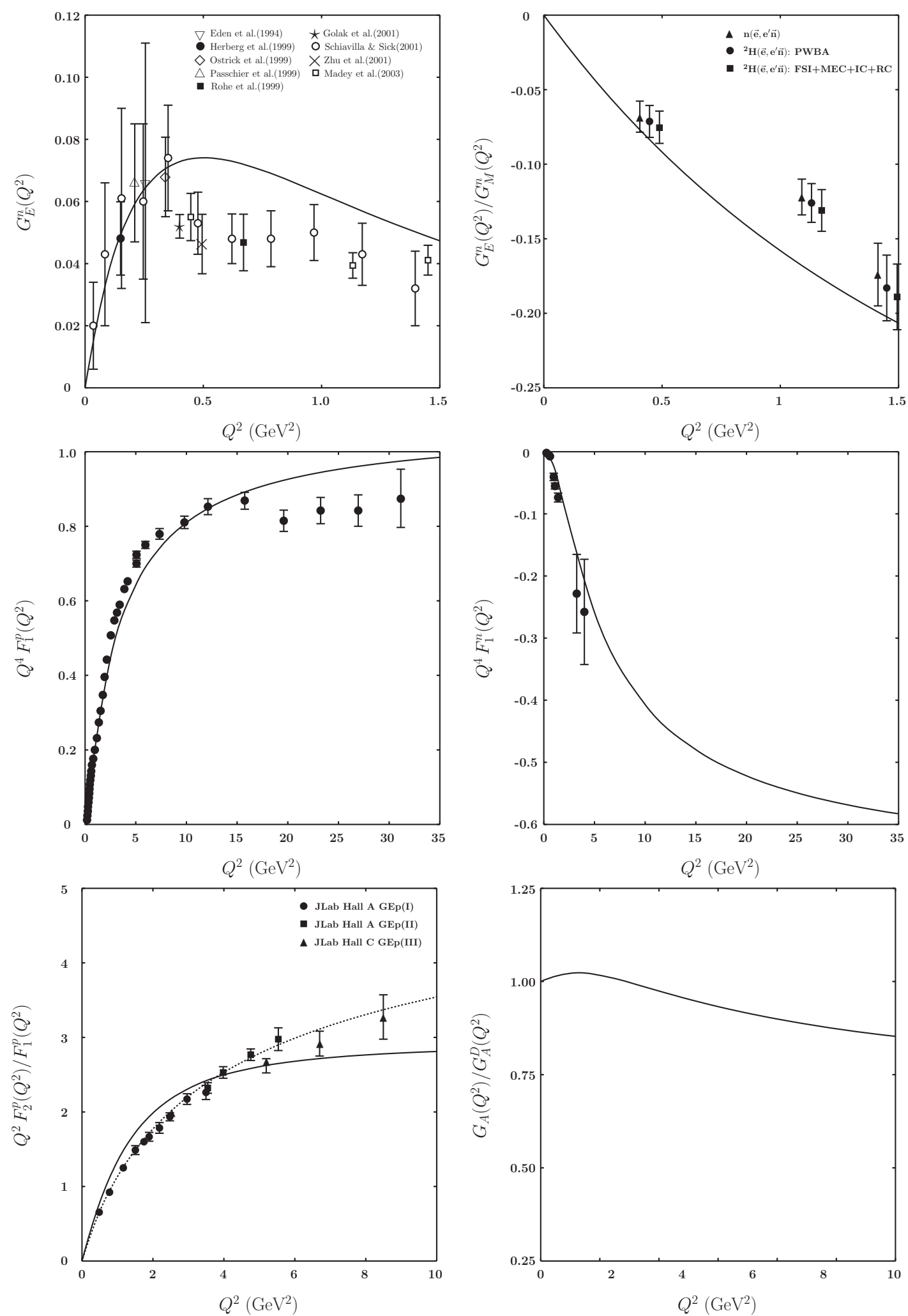

Figure 2: The charge neutron form factor, ratio $G_{E}^{n}\left(Q^{2}\right) / G_{M}^{n}\left(Q^{2}\right)$, proton and neutron Dirac form factor multiplied with $Q^{4}$, ratios $Q^{2} F_{2}^{p}\left(Q^{2}\right) / F_{1}^{p}\left(Q^{2}\right)$ (the dashed line is the approximation of data suggested in Ref. [11]) and $G_{A}\left(Q^{2}\right) / G_{A}^{D}\left(Q^{2}\right)$. 